\title{
Correction to: Rio: a personal storage system in multi-device and cloud
}

\section{Feng Wang ${ }^{1,3} \cdot$ Yanjun $\mathrm{Wu}^{1} \cdot$ Feiyue Huang ${ }^{2}$}

Published online: 12 May 2020

๑) Springer Science+Business Media, LLC, part of Springer Nature 2020

\section{Correction to: The Journal of Supercomputing (2020) 76:2315-2338 https://doi.org/10.1007/s11227-018-2501-8}

Dr. Feng Wang's second affiliation is missing in the original article. Both affiliations are given here.

The original article can be found online at https://doi.org/10.1007/s11227-018-2501-8.

Feng Wang

wangfeng@iscas.ac.cn

Yanjun Wu

yanjun@iscas.ac.cn

Feiyue Huang

huangfeiyue@gmail.com

1 Institute of Software, Chinese Academy of Sciences, Beijing, China

2 Tencent Youtu Lab, Shanghai, China

3 University of Chinese Academy of Sciences, Beijing, China 